\title{
Design and Implementation of OFDM Signal Processing on PSoC Microcontroller
}

\author{
Kohei Hayashi, Fumihito Sasamori, Osamu Takyu, and Shiro Handa \\ Shinshu University, 4-17-1 Wakasato, Nagano-shi, Nagano 380-8553, Japan \\ E-mail: 12tm242g@shinshu-u.ac.jp
}

\section{INTRODUCTION}

In recent years, the depletion of frequency band is a problem with the spread of smart phone, then orthogonal frequency division multiplexing (OFDM) systems have been attracting attention because of those spectral efficiency. OFDM systems have a feature that the frequency band can be efficiently utilized. This study has led to the development of low cost systems that use an OFDM signal and aims at implementation on a variety of products. We design and implement the OFDM signal on a Programmable System-on-Chip (PSoC) microcontroller which is easy-to-use, but has the disadvantage of limited hardware. In this report, we investigate a transmission of the OFDM signal with the PSoC microcontroller over voice-grade channels with free of charge in Japan.

\section{PSOC MICROCONTROLLER}

This study adopts the PSoC5 microcontroller made by the Cypress Semiconductor company. This microcontroller can operate both analog and digital circuits on software. A conventional microcontroller is a fixed module, where PSoC microcontroller can change the configuration of the module according to the purpose, whenever necessary. Since one can configure the analog circuits such as Analog to Digital Converter (ADC) and Digital Analog Converter (DAC) on PSoC microcontroller, an OFDM transmitter and receiver can be implemented on just one chip.

\section{SYSTEM OUTLINE}

In this paper, we assume voice-grade channels like Personal Handy-phone Systems (PHS) and cellular phone systems as a transmitter channel. Then we need to generate the OFDM signal with voice band of $300 \sim 3400 \mathrm{~Hz}$. Table 1 shows the other system specifications.

\section{A. System model}

Figure 1 shows the system model. Tx Data are fed into the digital modulation block, the Hermite-symmetric subcarrier coded OFDM (HC-OFDM) block [1], then the OFDM signal

TABLE I

SYSTEM SPECIFICATIONS.

\begin{tabular}{c|c}
\hline \hline Digital Modulation @ & QPSK \\
\hline OFDM Modulation & HC-OFDM \\
\hline Number of FFT Points & 64 \\
\hline Number of Subcarriers & 64 \\
\hline Guard Interval (GI) & 4 \\
\hline Quantization @ & 8 -bit \\
\hline Oversampling Rate & 6 \\
\hline Symbol Sampling Rate & 25000 sample/sec \\
\hline Sensing Rate & $80000 \mathrm{sample} / \mathrm{sec}$ \\
\hline \hline
\end{tabular}

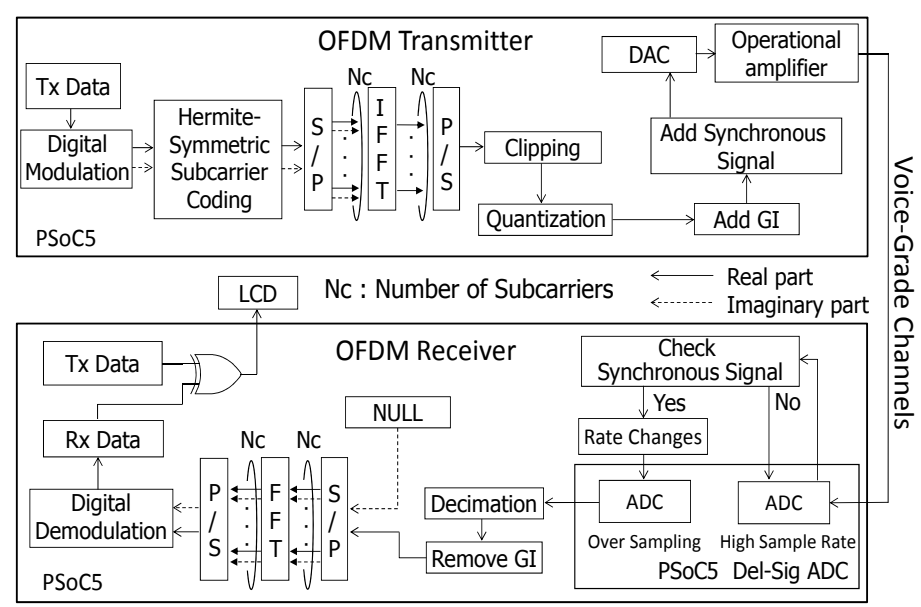

Fig. 1. System model.

without the imaginary part is generated. Hermite-symmetric subcarrier coding is based on the fact that the Fourier transform of real-valued input without imaginary part becomes Hermite-symmetric. Therefore, HC-OFDM is suitable for baseband transmission like asymmetric digital subscriber line (ADSL) and optical wireless communication.

The OFDM signal has large peak to average power ratio (PAPR) characteristics. Clipping the OFDM signal, that is, suppressing the maximum amplitude value helps reduce the PAPR easily. The clipped OFDM signal is quantized, added guard interval (GI), and added pilot signal mentioned later, then the desired OFDM signal can be output via DAC and operational amplifier (Op-Amp) for voltage follower. Figure 2 shows an example of the OFDM signal waveform. An impulse signal is used as the pilot signal to detect the OFDM symbol timing by the threshold detector at a receiver.

At the OFDM receiver, the signal is received through the ADC. Switching sampling rate of the ADC to high (which we call 'sensing rate' in Table 1) enables the symbol timing to be detected exactly. After synchronization, switching the sampling rate to low (which we call 'symbol sampling rate') and decimation enable the OFDM sample to be detected exactly. Rx Data are obtained by removing GI, HC-OFDM demodulation, and digital demodulation.

\section{B. OFDM signal design}

We set the symbol sampling rate to be 25000 sample per second at the receiver in Table 1 . In this section, we will explain the reason. First of all, we decide the transmission frequency band to be $300 \sim 3400 \mathrm{~Hz}$ and the oversampling 


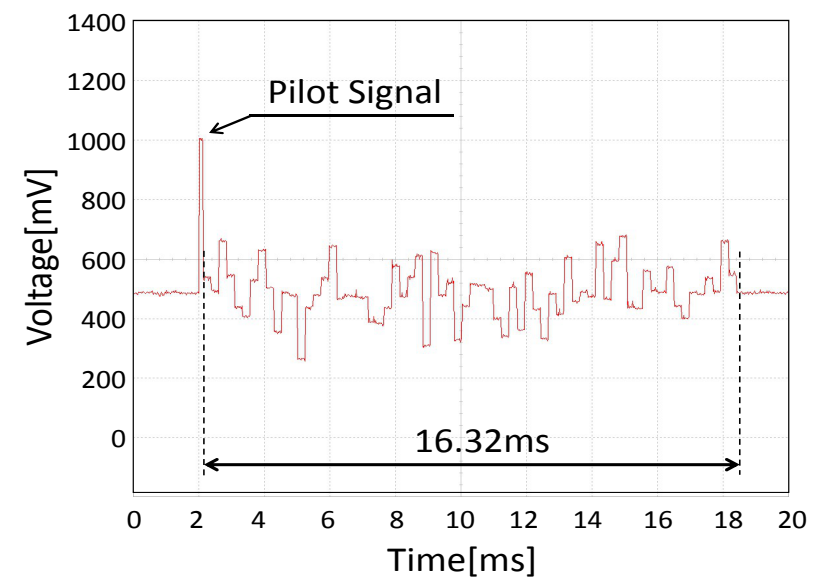

Fig. 2. Example of OFDM signal waveform.
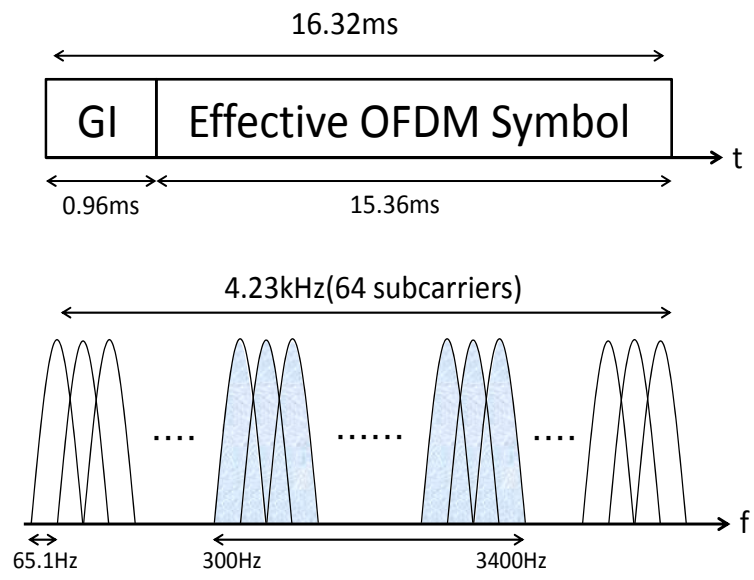

Fig. 3. Design of OFDM signal and its spectrum.

rate to be 6 . The rate of each OFDM sample which is input into 64-point FFT becomes 25000/6 sample per second, and then the interval between samples becomes 0.24 milliseconds (ms).

Figure 3 illustrates the OFDM signal and its spectrum. The GI duration becomes $0.96 \mathrm{~ms}$ (4 samples) and the effective OFDM symbol duration becomes $15.36 \mathrm{~ms}$ (64 samples). The spacing between adjacent subcarriers depends upon the effective OFDM symbol duration, then becomes $65.1 \mathrm{~Hz}$ $(=1 / 15.36 \mathrm{~ms})$. Therefore, the bandwidth of all 64 subcarriers becomes $4.23 \mathrm{kHz}$. Finally, we select the subcarriers within $300 \sim 3400 \mathrm{~Hz}$ and the others is not used (null-subcarrier).

\section{IMPROVEMENT OF TRANSMITTER}

In the current system, because of the limited hardware in PSoC, time delay caused by the computation has led to decrease the transmission rate. Just a PSoC microcontroller can not simultaneously transmit and receive data when modulating and demodulating the OFDM signal because of a single chip. Figure 4 illustrates the timing chart. No signal transmits during the modulation and demodulation, which decrease the transmission rate. This problem can be solved by linking multiple PSoC microcontrollers. Figure 5 shows the OFDM

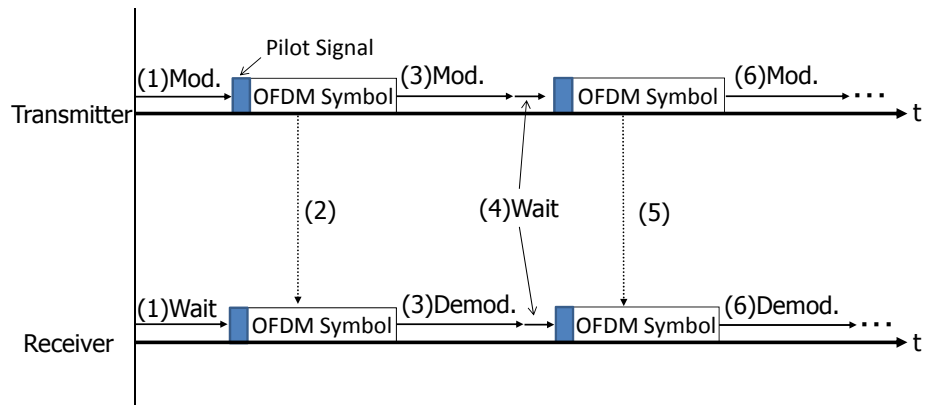

Fig. 4. Timing chart.

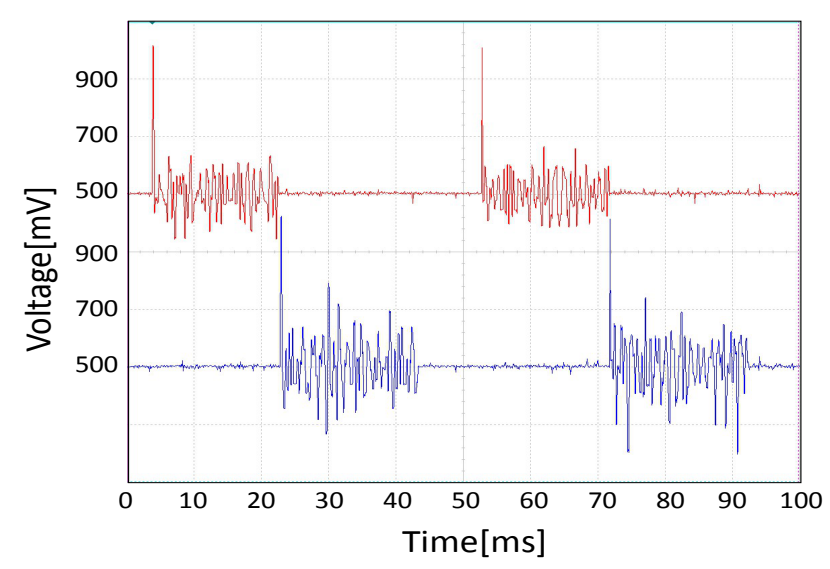

Fig. 5. OFDM signal waveform from two PSoC microcontrollers.

signal waveform from the transmitter that links two PSoC microcontrollers. The upper waveform is output from the first PSoC microcontroller, and the lower is from the second. The two alternately generate and transmit the OFDM signal. From the figure, we are able to nearly eliminate the delay caused by computation owing to linking multiple microcontrollers.

\section{CONCLUSION}

In this paper, we describe the construction of OFDM transmission system that implements the OFDM signal processing to PSoC5 microcomputer. In the future, we aim to build the receiving system that corresponds to the transmitter with multiple microcontrollers. Examining multi-level digital modulation causes to increase the amount of data per OFDM signal, then can improve the transmission rate. Moreover, we would like to design and implement the OFDM systems on PSoC microcontroller over several wireless channels.

\section{ACKNOWLEDGEMENT}

This work was supported by JSPS KAKENHI Grant Number 24560448 .

\section{REFERENCES}

[1] F.Sasamori and S. Handa "Performance Analysis of Hermite-Symmetric Subcarrier Coding for OFDM Systems over Fading Channels," IEICE Transactions on Fundamentals, vol.E95-A, no.12, pp.2461-2469, Dec. 2012 . 